\title{
Novel poly(ester amide)s from glycine and L-lactic acid by an easy and cost-effective synthesis
}

\author{
Ana C. Fonseca, ${ }^{a *}$ Arménio C. Serra, ${ }^{b}$ Jorge F.J. Coelho, ${ }^{a}$ Maria H. Gila \\ and Pedro N. Simões ${ }^{a}$
}

\begin{abstract}
Novel low molecular weight poly(ester amide)s based on glycine and L-lactic acid with interest for the biomedical field were successfully prepared by interfacial polymerization, which is an easy and fast polymerization method. Preparation of the $\alpha$-amino acid based diamine, the L-lactic acid based diacyl chlorides and the poly(ester amide)s was carried out in the absence of catalysts. The structure of the different poly(ester amide)s was confirmed by ${ }^{1} \mathrm{H}$ NMR and Fourier transform infrared spectroscopies. The thermal behaviour of the synthesized poly(ester amide)s was evaluated by simultaneous thermal analysis, differential scanning calorimetry and dynamic mechanical thermal analysis. It was found that both the incorporation of an L-lactic acid oligomeric segment and the change in its central unit have an important influence on the thermal characteristics of the poly(ester amide)s. These novel poly(ester amide)s can be used as building blocks for the preparation of more complex structures.
\end{abstract}

(c) 2013 Society of Chemical Industry

Supporting information may be found in the online version of this article.

Keywords: biomaterials; glycine; L-lactic acid; polycondensation; poly(ester amide)s

\section{INTRODUCTION}

Poly(ester amide)s (PEAs) are a class of synthetic polymers with increasing importance in the biomedical field, since they represent a good alternative to overcome the thermal and mechanical limitations of poly(lactic acid) (PLA) and poly(lactic-co-glycolic acid). ${ }^{1,2}$ PEAs gather in the same entity the best properties of polyesters (biodegradability) and polyamides (good mechanical and thermal behaviour). ${ }^{2,3}$ PEAs containing $\alpha$-amino acids and $\alpha$-hydroxy acids in their structure (e.g. L-lactic acid (L-LA) or glycolic acid), commonly known as polydepsipeptides, also demonstrated their potential in biomedical applications as devices for drug delivery systems and scaffolds for tissue engineering. ${ }^{4-6}$ Upon metabolic degradation, these PEAs release $\alpha$-amino acids and $\alpha$-hydroxy acids which are common metabolites in the human body, and thus the toxicity induced by this type of materials is very low. Considering that the degradation products have both acid and basic natures, an in situ neutralization reaction occurs, thus preventing accentuated $\mathrm{pH}$ decrease, which is typical in polyester degradation. ${ }^{7}$ Moreover, the presence of the $\alpha$-amino acid in the polymer structure opens up the possibility of their enzymatic degradation contributing to the high biodegradability of PEAs compared with polyesters. ${ }^{8,9}$ The most common method for PEA synthesis is based on the ring-opening polymerization of morpholine-2,5-dione derivatives. ${ }^{4,10,11}$ Although it is the most commonly used method, it was observed that low molecular weight oligomers with poor mechanical and thermal properties are formed in some cases. Additionally, morpholine-2,5-dione derivatives are obtained in relatively low yields (ca 30\%). ${ }^{12}$
In this work, PEAs based on glycine and L-LA were obtained by interfacial polymerization, using a new diamine derivative from glycine and a diacyl chloride derived from L-LA oligomers. Interfacial polymerization is a fast and easily scalable method in which the polymer is obtained in a few hours of reaction (3 h). ${ }^{13}$ The preparation of PEAs through the use of L-LA derived diacyl chlorides, together with the use of both conventional diamines and $\alpha$-amino acid based diamines, has been reported in the literature. D'Angelo et al. ${ }^{14}$ proposed the synthesis of PEAs based on glycine and L-phenylalanine by this approach. However, the synthetic route to obtain diamines from $\alpha$-amino acids is quite troublesome, involving several protection/deprotection steps of the $\alpha$-amino acid functional groups. In our easier and straightforward strategy, a new diamine monomer was obtained by Fischer esterification in the presence of $p$-toluene sulfonic acid monohydrate. This method has been widely used for the synthesis of this type of monomers. ${ }^{7,12,15}$ It is worth mentioning that the glycine based diamine monomer was synthesized for the first time. This new glycine based diamine monomer has ether linkages that will influence important properties of the polymer,

\footnotetext{
* Correspondence to: Ana C. Fonseca, Department of Chemical Engineering, University of Coimbra, Rua Sílvio Lima-Pólo II, 3030-290 Coimbra, Portugal. E-mail:anafs@eq.uc.pt
}

a Department of Chemical Engineering, University of Coimbra, Rua Sílvio Lima-Pólo Il, 3030-290, Coimbra, Portugal

b Department of Chemistry, University of Coimbra, 3004-535, Coimbra, Portugal 
such as hydrophilicity, chain flexibility and biodegradability. ${ }^{16}$ Additionally, the L-LA oligomer precursors for preparation of the diacyl chlorides were synthesized from the L-LA monomer, rendering the synthesis less expensive than those in which the Llactide is used. Another advantage of our method is that no catalyst was used, thus avoiding troublesome procedures to remove it at the end of the polymerization.

\section{EXPERIMENTAL}

\section{Materials}

L-LA (80\%), ethylene glycol (EG) (99\%), diethylene glycol (DEG) (99\%), poly(ethylene glycol) (PEG, $\left.300 \mathrm{~g} \mathrm{~mol}^{-1}\right), p$ toluenesulfonic acid monohydrate ( $p$-TSA), glycine (>99\%), sebacoyl chloride (92\%), anhydrous sodium carbonate $\left(\mathrm{Na}_{2} \mathrm{CO}_{3}\right)$, $\mathrm{N}, \mathrm{N}$-dimethylformamide (HPLC grade) and chloroform (HPLC grade) were purchased from Sigma-Aldrich (St Louis, MO, USA) and used as received. Dichloromethane, toluene and acetone were supplied by José Manuel Gomes dos Santos Lda (Odivelas, Portugal) and were purified by standard methods before use. Deuterated dimethylsulfoxide (DMSO- $\mathrm{d}_{6}$ ) was purchased from Eurisotop (Saint-Aubin, France).

\section{Measurements}

The elemental composition of the bis( $\alpha$-amino acid) ester was determined using an elemental analyser EA1108 CHNS-O from Fisons Instruments.

The number-average $\left(M_{n}\right)$ and weight-average $\left(M_{\mathrm{w}}\right)$ molecular weight of LA oligomers were determined by SEC using two PLgel Mixed-D columns (Polymer Laboratories) coupled to an evaporative light scatttering detector, PL-EMD 960. Chloroform was used as eluent and the system was calibrated with narrow polystyrene standards.

The molecular weight distribution of the PEAs was determined using an HPSEC Viscotek (Dual detector 270, Viscotek, Houston, TX, USA) with a differential viscosimeter detector and refractive index detector (Knauer K-2301). The column set consisted of a PL $10 \mu \mathrm{m}$ guard column $(50 \mathrm{~mm} \times 7.5 \mathrm{~mm})$ followed by two Mixed-B PL columns $(300 \mathrm{~mm} \times 7.5 \mathrm{~mm}, 10 \mu \mathrm{m})$. The HPLC pump (Knauer $\mathrm{K}-1001$ ) was set with a flow rate of $1 \mathrm{~mL} \mathrm{~min}{ }^{-1}$. The system was also equipped with a Knauer on-line degasser. The tests were done at $60{ }^{\circ} \mathrm{C}$ using an Elder $\mathrm{CH}-150$ heater. $N, N^{\prime}$-dimethylformamide with $\mathrm{LiBr}(0.03 \% \mathrm{w} / \mathrm{w})$ was used as the eluent. Universal calibration was done with four narrow polystyrene standards $\left(M_{p}\left(\mathrm{~g} \mathrm{~mol}^{-1}\right)=\right.$ 1820, 4290, 10 050, 30 300).

Fourier transform infrared (FTIR) spectra were obtained in the range $4000-500 \mathrm{~cm}^{-1}$ at room temperature using a Jasco FT/IR4200 spectrometer equipped with a Golden Gate Single Reflection Diamond ATR. Data collection was performed with $4 \mathrm{~cm}^{-1}$ spectral resolution and 128 accumulations. ${ }^{1} \mathrm{H}$ NMR spectra were obtained at $25^{\circ} \mathrm{C}$ on a Varian Unity $600 \mathrm{MHz}$ spectrometer using a $3 \mathrm{~mm}$ broadband NMR probe, in DMSO- $\mathrm{d}_{6}$. Tetramethylsilane was used as internal standard.

The thermal stability of the PEAs was first evaluated by simultaneous thermal analysis (heat-flux DSC and TGA), using TA Instruments SDT Q600 equipment (thermobalance sensitivity $0.1 \mu \mathrm{g})$, which was previously calibrated in the range $25-1000^{\circ} \mathrm{C}$ by running tin and lead as melting standards, at a heating rate $(\phi)$

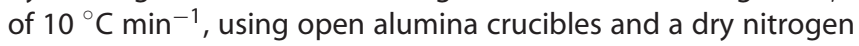
purge flow of $100 \mathrm{~mL} \mathrm{~min}^{-1}$. Sample weights ranging from 8 to 10 $\mathrm{mg}$ were used.
The thermal behaviour of the PEAs was further studied by modulated differential scanning calorimetry (MDSC) in a TA Instruments Q100 model equipped with an RCS90 cooling unit. The heat flow and the heat capacity were calibrated at $2{ }^{\circ} \mathrm{C} \mathrm{min}^{-1}$ using, respectively, indium and sapphire standards. The samples were analysed in aluminium pans with an ordinary aluminium lid loosely placed. Sample weights ranging from 5 to $10 \mathrm{mg}$ were used. A heating rate of $2{ }^{\circ} \mathrm{C} \mathrm{min}-1$, a modulation period of $60 \mathrm{~s}$ and a temperature modulation of $\pm 0.40{ }^{\circ} \mathrm{C}$ were applied. A dry

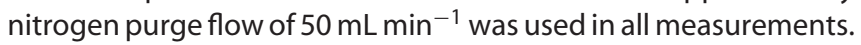
For glass transition temperature measurements, the samples were heated at $2{ }^{\circ} \mathrm{C} \mathrm{min}-1$ from -85 to $190{ }^{\circ} \mathrm{C}$, after performing a cycle in which the samples were heated from $25{ }^{\circ} \mathrm{C}$ to $190{ }^{\circ} \mathrm{C}$ and cooled to $-85^{\circ} \mathrm{C}$ to erase the samples' thermal history. The values of the glass transition temperature were obtained from the corresponding heat capacity step (half-height), obtained from the reversing signal.

Dynamic mechanical thermal analysis (DMTA) was carried out using a Tritec 2000 DMA. The runs were performed using PEAs in powder form, placed in stainless steel material pockets, in single cantilever bending geometry. The tests were carried out from $-150{ }^{\circ} \mathrm{C}$ to $190^{\circ} \mathrm{C}$, in multifrequency mode, with a heating rate of $2^{\circ} \mathrm{C} \min ^{-1}$.

\section{Synthesis of LA telechelic oligomers}

For the preparation of L-LA oligomers with - $\mathrm{OH}$ terminal groups, L-LA was copolymerized with EG (PLA-OH 1) or PEG (PLA-OH 2) (10 mol\% relative to the amount of L-LA). The polymerization occurred in the melt for $13 \mathrm{~h}$, at atmospheric pressure and $170{ }^{\circ} \mathrm{C}$ (Scheme 1). During the reaction, a continuous stream of nitrogen was maintained under the surface of the melt. ${ }^{17,18}$ The oligomers were purified by a dissolution (acetone)/precipitation (water) method. The excess solvent was removed under vacuum, and the oligomer was placed in a vacuum oven at $40^{\circ} \mathrm{C}$. PLA-OH 1 , $M_{\mathrm{n}}=598 \mathrm{~g} \mathrm{~mol}^{-1}, M_{\mathrm{w}} / M_{\mathrm{n}}=1.32 ;$ PLA-OH $2, M_{\mathrm{n}}=600 \mathrm{~g} \mathrm{~mol}^{-1}$, $M_{\mathrm{w}} / M_{\mathrm{n}}=1.18$.

\section{Synthesis of bis ( $\alpha$-amino acid) ester from glycine and DEG (1)} A suspension of glycine (27.5 mmol), DEG (12.5 mmol) and $p$-TSA (30 mmol) in toluene (Scheme 2) was heated to $130{ }^{\circ} \mathrm{C}$ with stirring, in a round bottom flask equipped with a Dean-Stark<smiles>CC(O)C(=O)[OH+][CH+]OCC=O</smiles><smiles>CC(O)C(=O)OC(C)C(=O)OCCOC(=O)C(C)OC(=O)C(C)OC(=O)C(C)O</smiles>

PLA-OH1

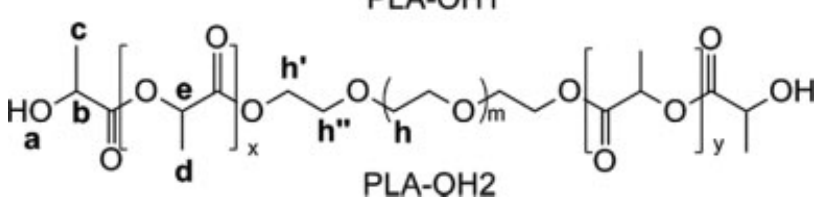

Scheme 1. Reaction between L-LA and EG and L-LA and PEG. 
<smiles>NCC(=O)O</smiles>

(1)

Scheme 2. Reaction between glycine and DEG.

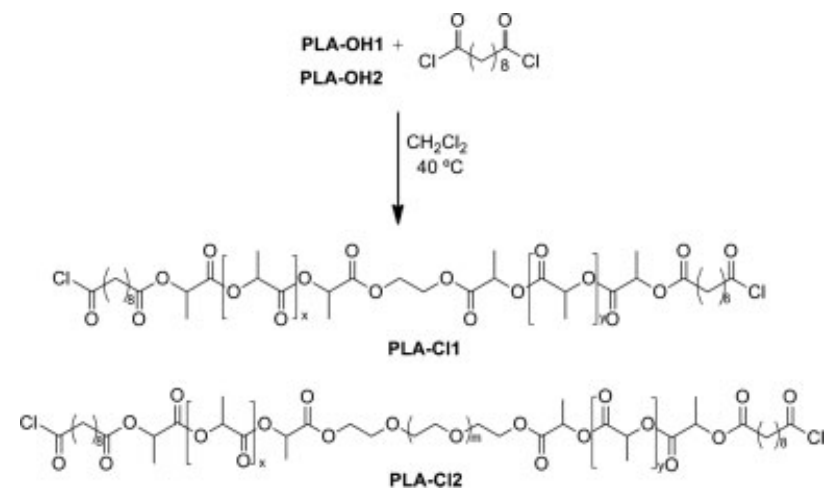

Scheme 3. Reaction between L-LA telechelic oligomers and sebacoyl chloride.

apparatus and a condenser with a drying tube. The suspension was heated to reflux until no more water was distilled. The excess toluene was removed under vacuum and the resulting material was recrystallized from an isopropanol/ethyl acetate mixture $(80 / 20, v / v) .^{19,20}$

The yield was $48 \%$. Melting point $116-120{ }^{\circ} \mathrm{C}$. Analysis calculated for $\mathrm{C}_{22} \mathrm{H}_{32} \mathrm{~N}_{2} \mathrm{O}_{11} \mathrm{~S}_{2}, \mathrm{C} 46.80, \mathrm{H}$ 5.67, $\mathrm{N}$ 4.96; found, C 46.24, H 5.61, N 5.19. IR (ATR): 1756 ( $\left(\mathrm{C}=\mathrm{O}_{\text {ester }}\right), 1212$ and $1120\left(v \mathrm{C}-\mathrm{O}-\mathrm{C}_{\text {ester }}\right), 1030$ and $1010 \mathrm{~cm}^{-1}$ (vas $\mathrm{SO}_{3}{ }^{-}$, vs $\mathrm{SO}_{3}{ }^{-}$, respectively). ${ }^{1} \mathrm{H}$ NMR $\left(600 \mathrm{MHz}, \mathrm{DMSO}-\mathrm{d}_{6}\right): \delta=7.49-7.13\left(\mathrm{~d}, \mathrm{H}^{8}\right.$, $\mathrm{Ar}-\mathrm{H}), 4.28\left(\mathrm{t}, \mathrm{H}^{4}, \mathrm{COOCH}_{2} \mathrm{CH}_{2}\right), 3.85\left(\mathrm{~s}, \mathrm{H}^{4}, \mathrm{NH}_{3}{ }^{+} \mathrm{CH}_{2}\right), 3.68\left(\mathrm{t}, \mathrm{H}^{4}\right.$, $\left.\mathrm{COOCH}_{2} \mathrm{CH}_{2}\right), 2.29\left(\mathrm{~s}, \mathrm{H}^{6}, \mathrm{Ar}-\mathrm{CH}_{3}\right)$.

\section{Synthesis of diacyl chlorides from L-LA oligomers}

The L-LA telechelic oligomers (PLA-OH 1 or PLA-OH 2) (3 mmol) were dissolved in dichloromethane $(3.5 \mathrm{~mL})$ and added dropwise to a stirred solution of sebacoyl chloride $(6 \mathrm{mmol})$ in dichloromethane (3.5 mL), at $40{ }^{\circ} \mathrm{C}^{14,21}$ The reaction proceeded for $2 \mathrm{~h}$ (Scheme 3), yielding L-LA based diacyl chlorides, PLA-Cl 1 and PLA-Cl 2. This organic solution was used directly in the preparation of the PEAs.

\section{Synthesis of PEAs}

Interfacial polymerization of 1 with PLA-Cl 1 and PLA-Cl 2.

Compound 1 ( $3 \mathrm{mmol}$ ) was dissolved in distilled water $(9 \mathrm{~mL})$ and $\mathrm{Na}_{2} \mathrm{CO}_{3}(6 \mathrm{mmol})$ was added. The resulting solution was placed in an ice-bath. Then the organic solution containing PLA-Cl 1 or PLA$\mathrm{Cl} 2$ was added dropwise to the aqueous solution under stirring. The reaction proceeded for $2 \mathrm{~h}$ (Scheme 4). ${ }^{19,20}$ The precipitated polymer was recovered by filtration, washed thoroughly with acetone and distilled water and dried under vacuum at $40{ }^{\circ} \mathrm{C}$.

Interfacial polymerization of $\mathbf{1}$ with sebacoyl chloride.

A solution of sebacoyl chloride $(3 \mathrm{mmol}$ ) in dichloromethane $(9 \mathrm{~mL})$ was added dropwise to a stirred aqueous solution of 1 (3 mmol) and $\mathrm{Na}_{2} \mathrm{CO}_{3}(6 \mathrm{mmol})$. After the addition was complete, stirring was continued for $2 \mathrm{~h}$ (Scheme 5) and the precipitated polymer was isolated by filtration, washed thoroughly with acetone and distilled water and dried under vacuum at $40{ }^{\circ} \mathrm{C}^{19,20}$

\section{RESULTS AND DISCUSSION}

The synthesis of L-LA oligomers with hydroxyl terminal groups was carried out in the melt, using the L-LA monomer in the presence of EG or PEG as comonomers (Scheme 1). The diols react with a molecule of L-LA or a small L-LA oligomeric chain, yielding oligomeric chains with hydroxyl terminal groups. As the polymerization proceeds, the carboxylic groups of the L-LA monomer or the L-LA oligomeric chain react with the hydroxyl groups of the telechelic chain. As a result, the product at the end of the polymerization presents only one kind of terminal group. ${ }^{17,18}$

FTIR spectra of L-LA oligomers (Fig. 1 and Table 1) showed the bands corresponding to the hydroxyl terminal groups as well as ester and ether linkages.

The ${ }^{1} \mathrm{H}$ NMR spectrum allowed the identification of the resonances of the L-LA oligomer central units and those corresponding to the terminal hydroxyl groups (Fig. 2; see Scheme 1 for atom labelling). The spectroscopic data concerning the L-LA oligomers with hydroxyl terminal groups are in accordance with the anticipated chemical structure. At $\delta \approx 4.03 \mathrm{ppm}\left({ }^{*}\right)$, a quartet is detected that can be attributed to unreacted L-LA monomer. The residual monomer is about $8 \%$ (determined from the ratio of the integrals of signals e and ${ }^{*}$ ) of the total L-LA oligomeric mixture. Since the L-LA oligomers will react with sebacoyl chloride to form a macrodiacyl chloride, the occurrence of side reactions between the unreacted L-LA and sebacoyl chloride cannot be totally excluded (acylation of the - $\mathrm{OH}$ group of L-LA or formation of a mixed anhydride) at this point. This will decrease the amount of acyl functionalities available to react with the diamine in the subsequent reaction steps, leading to a decrease in PEA molecular weight. This will be appropriately discussed below.

The PEAs (PEA 1 and PEA 2) were synthesized by interfacial polymerization of PLA-Cl 1 and PLA-Cl 2 with 1 (Scheme 4). For comparison, a PEA with sebacoyl chloride and 1 (PEA 3) was prepared (Scheme 5). The aim underlying the preparation of different PEAs was twofold: to understand the influence of the incorporation of an oligomeric L-LA chain on the PEA properties, and to evaluate the effect of the L-LA chain central unit. FTIR spectra of PEAs (Fig. 3) clearly show the characteristic bands of ester and amide linkages, which are listed in Table 2.

In the FTIR spectra of PEA 1 and PEA 2, a shoulder at ca $1700 \mathrm{~cm}^{-1}$, which can be attributed to the $v C=0$ of free carboxylic groups, is observable. This shoulder vanishes in the spectrum of PEA 3. This suggests the presence of sebacic acid, an issue that needs to be further discussed. The hydrolysis of sebacoyl chloride leads to the formation of sebacic acid, but this compound can also be formed in the reaction between the mixed anhydride (resultant from the reaction of unreacted L-LA oligomer with sebacoyl chloride) and the amino groups in the glycine based diamine. Thus, two main 


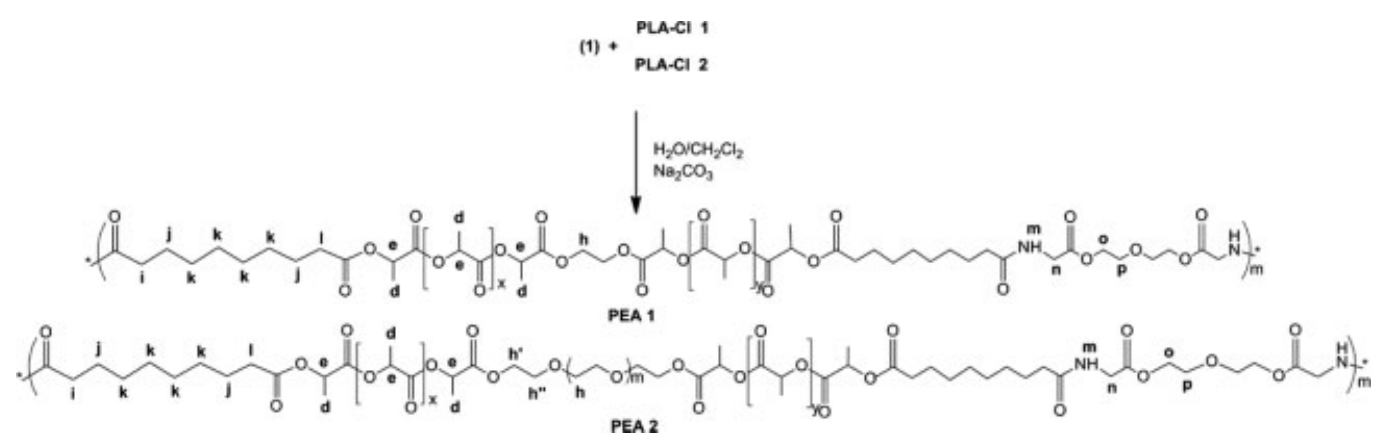

Scheme 4. Reaction between 1 and L-LA derived diacyl chlorides.

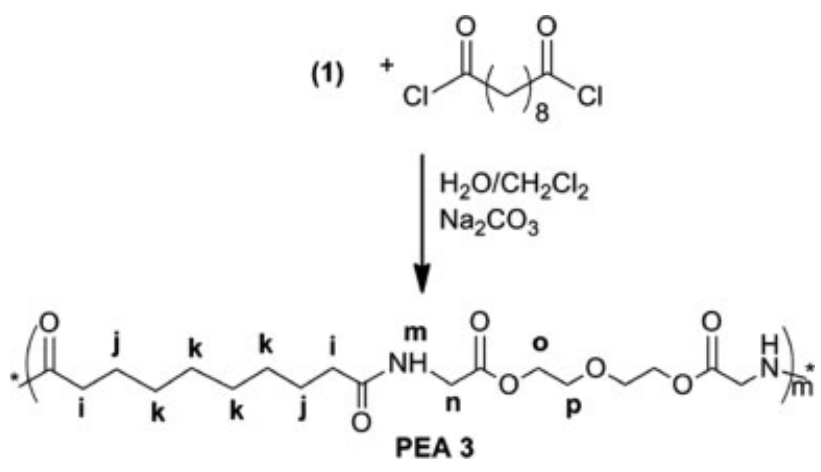

Scheme 5. Reaction between $\mathbf{1}$ and sebacoyl chloride.

factors are responsible for the shortage of acyl functionalities of the L-LA macrodiacyl chlorides: sebacoyl chloride hydrolysis and the presence of the mixed anhydride.

The comparative ${ }^{1} \mathrm{H}$ NMR spectra of the PEAs are presented in Fig. 4 (see Schemes 4 and 5 for atom labelling). The resonance of the amide proton is clearly visible at $\delta \approx 8.25 \mathrm{ppm}$. The resonances in the 5.2-4.96 ppm range are attributed to the $-\mathrm{CH}$ groups of $\mathrm{L}-\mathrm{LA}$ units. In the 4.1-3.7 ppm range it is possible to observe the resonances of the $-\mathrm{CH}_{2}$ protons of the EG or PEG linked to the L-LA oligomeric chain. In PEA 1 and PEA 2, the two distinct resonances at $\delta \approx 2.36$ (I) and $2.14 \mathrm{ppm}$ (i) reflect the different chemical environments experienced by the external $-\mathrm{CH}_{2}$ protons of the sebacoyl group (on one side they are linked to an amide linkage (i) whereas on the other side they are linked to an ester linkage (I); see Fig. 3 for the PEA structure). As expected, this difference is not observed in PEA 3 since both $-\mathrm{CH}_{2}$ moieties are linked to an amide linkage.

As mentioned above, the unreacted L-LA monomer in the L-LA oligomeric mixture could participate in side reactions with sebacoyl chloride (acylation of the -OH group of L-LA or formation of a mixed anhydride). In the ${ }^{1} \mathrm{H}$ NMR spectra of PEA 1 and PEA 2 , the resonance corresponding to the external $-\mathrm{CH}_{2}$ protons of sebacic acid appears at $\delta \approx 2.18 \mathrm{ppm}\left({ }^{*}\right)$. This resonance is more intense in the ${ }^{1} \mathrm{H}$ NMR spectra of PEA 1 and PEA 2 than in the spectrum of PEA 3, which indicates the presence of higher

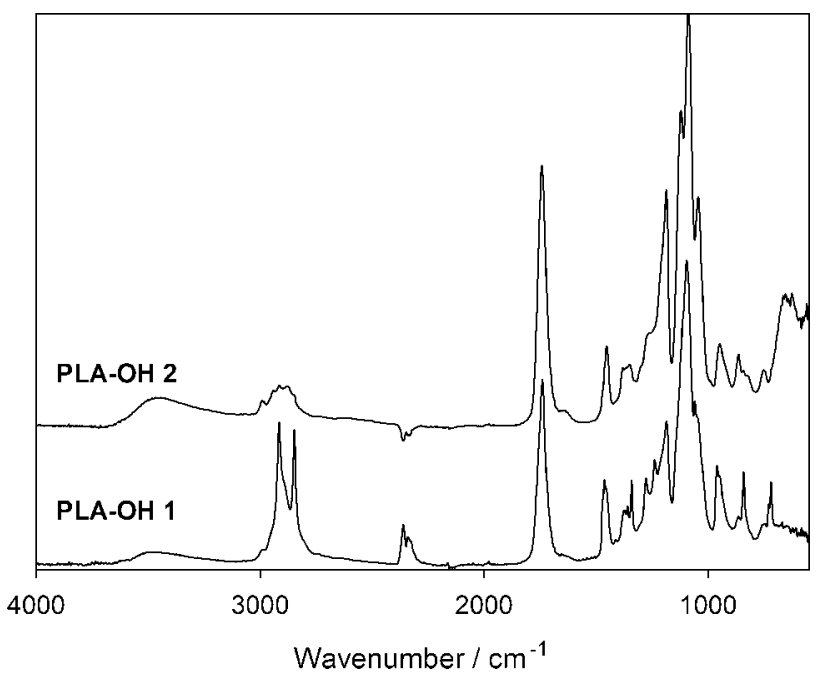

Figure 1. FTIR spectra of the L-LA oligomers with hydroxyl terminal groups.

amounts of sebacic acid, corroborating the results obtained in the FTIR spectroscopy analysis.

The molecular weight distribution (MWD) of the PEAs was measured by SEC (universal calibration) using the viscosity measured with the on-line viscosimeter. All the PEAs present a broad MWD (Fig. S1) with a polydispersity index of ca 2.5 and molecular weight between 2200 and $7400 \mathrm{~g} \mathrm{mo1}^{-1}$ (Table 3). PEA 1 and PEA 2 present a lower molecular weight than PEA 3, which is related to the lower amount of diacyl chloride present in the reaction medium, as discussed above.

Compared with the other methods available in the literature for the preparation of PEAs, the method reported here is faster, easier and is carried out in the absence of any catalyst. ${ }^{7,22,23}$ Nevertheless, the method needs to be optimized to afford a higher molecular weight and a narrow MWD. ${ }^{24}$

Simultaneous thermal analysis (TGA/DSC) (Fig. 5, Table 4) showed that the PEAs have a similar weight loss pattern, hence revealing that the incorporation of the LA oligomeric chains in

Table 1. Main IR bands and respective assignments of L-LA hydroxyl terminated oligomers

\begin{tabular}{lccccc|} 
& \multicolumn{4}{c}{ Infrared bands $\left(\mathrm{cm}^{-1}\right)$} \\
\cline { 2 - 6 } L-LA oligomer & $\nu \mathrm{O}-\mathrm{H}$ & $\nu \mathrm{C}=\mathrm{O}_{\text {ester }}$ & $\nu \mathrm{C}-\mathrm{O}-\mathrm{Cs}_{\text {ester }}$ & $\nu \mathrm{C}-\mathrm{O}-\mathrm{Cas}_{\text {ester }}$ & $\nu \mathrm{C}-\mathrm{O}-\mathrm{Cas}$ ether \\
\hline PLA-OH 1 & 3480 & 1740 & 1187 & 1096 & 1089 \\
PLA-OH 2 & 3446 & 1741 & 1187 & 108 & 1122 \\
\hline
\end{tabular}




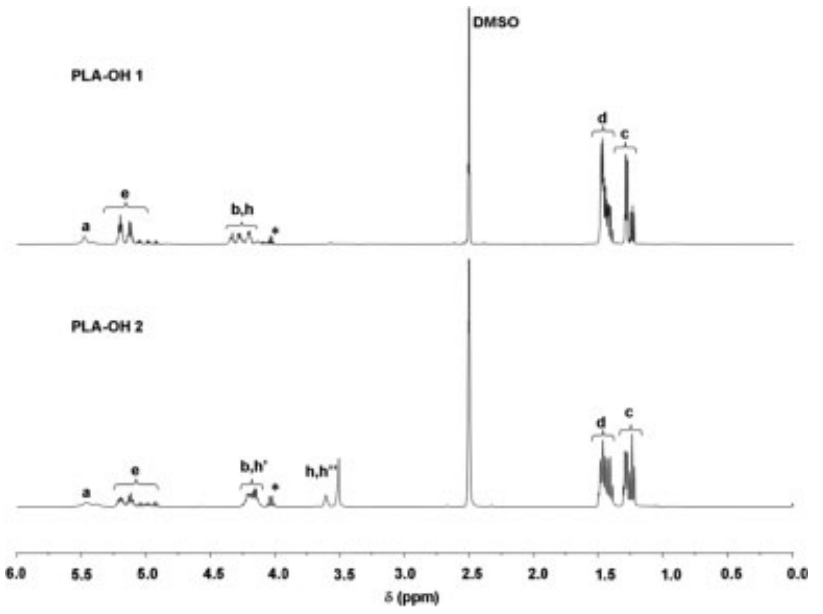

Figure 2. ${ }^{1} \mathrm{H}$ NMR spectra of L-LA oligomers with hydroxyl terminal groups.

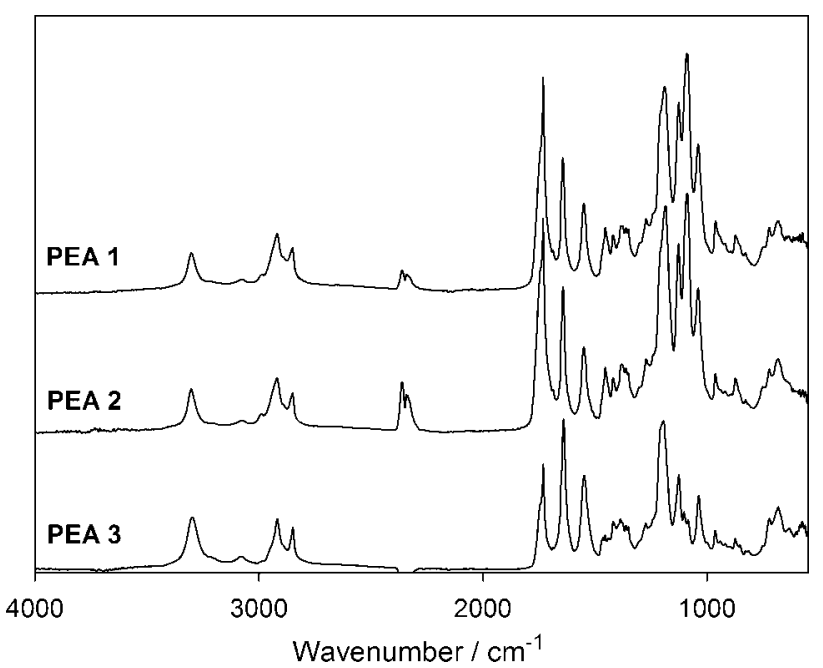

Figure 3. FTIR spectra of the synthesized PEAs.

the PEA structure does not negatively influence their thermal stability.

The small difference in the thermal stability can be ascribed mainly to the differences in the corresponding molecular weight (Table 4, $T_{\text {on }}$ ). The differential thermogravimetric (DTG) curves (Fig. 5(B)) evidence two distinct degradation stages. Armelin et al. ${ }^{8}$ observed the same degradation pattern in PEAs derived from $\alpha$-amino acids and sebacoyl chloride. Luckachan and Pillai ${ }^{2}$ synthesized PEAs containing poly(L-lactide) segments and cycloaliphatic amide segments and found that the thermal degradation also proceeded in two steps, the first being attributed to the degradation of

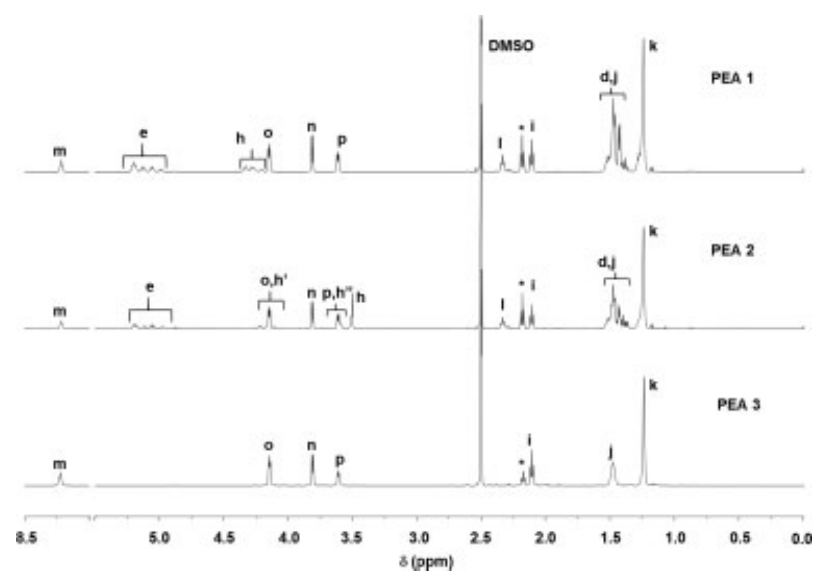

Figure 4. ${ }^{1} \mathrm{H}$ NMR spectra of the synthesized PEAs.

Table 3. Molecular weight $\left(\overline{M_{p}}\right)$ of the synthesized PEAs

\begin{tabular}{|lc|} 
Poly(ester amide) & $\overline{M_{p}}\left(\mathrm{~g} \mathrm{~mol}^{-1}\right)$ \\
\hline PEA 1 & 4006 \\
PEA 2 & 2238 \\
PEA 3 & 7388 \\
\hline
\end{tabular}

ester and the second to amide bond cleavage. Based on the available information, one can ascribe the first weight loss stage to the degradation of ester linkages along with ether linkages, whereas the second can be attributed to the degradation of the amide linkages. Concerning the thermal stability of ester and amide linkages, it has been reported that the amide linkages present higher stability than the ester linkages due to the partial double bond character of the $\mathrm{CN}$ bond. ${ }^{25}$ No additional weight loss is observed for lower temperatures; thus the formation of undesired sub-products or oligomers with low thermal stability is unlikely.

Thermal events in the PEAs below the degradation temperature were evaluated in more detail by MDSC and by DMTA. Figure 6 presents the reverse heat flow curves for the synthesized PEAs. All PEAs present a well defined endothermic peak above $120^{\circ} \mathrm{C}$ (all the temperatures mentioned hereafter correspond to peak temperatures). In PEA 1 and PEA 2 an additional and well defined peak appears at ca $85^{\circ} \mathrm{C}$. PEA 3 exhibits multiple weak endothermic peaks at $c a 88^{\circ} \mathrm{C}$ and $c a 106^{\circ} \mathrm{C}$. This is a common feature in PEAs, which resembles the thermal behaviour of polyamides, ${ }^{19,20,26}$ and is ascribed to the presence of different populations of lamellar crystals with different thicknesses. ${ }^{19,20}$ Similarly to what occurs with polyamides, in PEAs the smaller and imperfect crystals melt and tend to rearrange themselves into more stable lamellae. ${ }^{26,27}$ In PEA 3, it is possible to observe the absence of those multiple endothermic peaks in the second heating run, probably as a

Table 2. Main IR bands of PEAs and respective assignment of the synthesized PEAs

Infrared bands $\left(\mathrm{cm}^{-1}\right)$

\begin{tabular}{|lccccc|}
\cline { 2 - 5 } Poly(ester amide) & Amide $\mathrm{A}(\nu \mathrm{N}-\mathrm{H})$ & Amide $\mathrm{E}$ & Ester $(\nu \mathrm{C}=\mathrm{O})$ & Amide $\mathrm{I}(\nu \mathrm{C}=\mathrm{O})$ & Amide $I(\delta \mathrm{N}-\mathrm{H}$ and $\nu \mathrm{C}-\mathrm{N})$ \\
\hline PEA 1 & 3304 & 3078 & $1733(1700 \mathrm{sh} ; 1745 \mathrm{sh})$ & 1643 & 1550 \\
PEA 2 & 3302 & 3077 & $1732(1700 \mathrm{sh} ; 1743 \mathrm{sh})$ & 1644 & 1550 \\
PEA 3 & 3299 & 3083 & $1732(1743 \mathrm{sh})$ & 1640 & 1549 \\
\hline
\end{tabular}



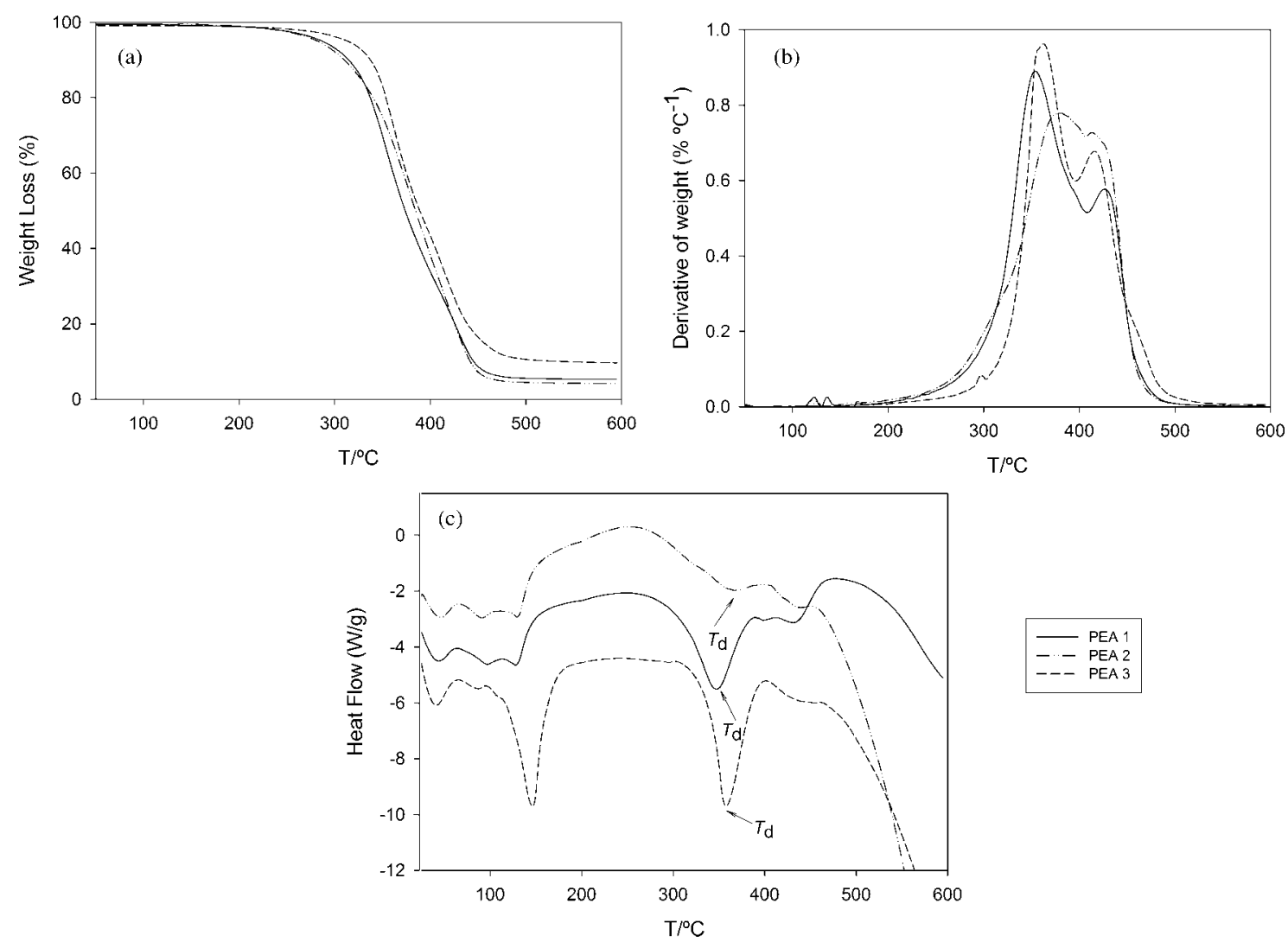

Figure 5. Thermoanalytical curves of PEAs at $10^{\circ} \mathrm{C} \mathrm{min}^{-1}$ : (A) TG; (B) DTG; (C) heat flow DSC.

\begin{tabular}{|c|c|c|c|c|c|}
\hline Poly(ester amide) & $T_{\text {on }}\left({ }^{\circ} \mathrm{C}\right)$ & $T_{10 \%}\left({ }^{\circ} \mathrm{C}\right)$ & $T_{\mathrm{p}, 1}\left({ }^{\circ} \mathrm{C}\right)$ & $T_{\mathrm{p}, 2}\left({ }^{\circ} \mathrm{C}\right)$ & $T_{\mathrm{d}}\left({ }^{\circ} \mathrm{C}\right)$ \\
\hline PEA 1 & $318.79 \pm 0.37$ & $315.92 \pm 1.91$ & $353.55 \pm 0.03$ & $426.51 \pm 0.42$ & $346.61 \pm 0.41$ \\
\hline PEA 2 & $322.65 \pm 0.75$ & $311.37 \pm 0.42$ & $382.44 \pm 2.30$ & $418.64 \pm 0.42$ & $362.25 \pm 4.6^{a}$ \\
\hline PEA 3 & $333.09 \pm 2.45$ & $336.92 \pm 0.37$ & $360.17 \pm 1.97$ & $415.12 \pm 0.99$ & $355.39 \pm 2.57$ \\
\hline
\end{tabular}

$T_{\text {on, }}$ extrapolated onset temperature (TG); $T_{10 \%}$, temperature corresponding to $10 \%$ mass loss; $T_{\mathrm{p}}$, peak temperature (DTG); $T_{\mathrm{d}}$, degradation temperature of the PEAs (DSC).

a Very broad peak which impairs an accurate determination of the $T_{\mathrm{d}}$ value.

consequence of a structure with more perfect crystals, with a melting temperature of $c a 140{ }^{\circ} \mathrm{C}$. All the PEAs have in common the segment containing the amide linkages (that corresponds to the repeating unit of PEA 3). The melting temperature of such segment corresponds to the higher temperature detected by MDSC. It should be pointed out that a decrease in this temperature is observed when the L-LA oligomeric segment is incorporated in PEAs (see Fig. 3 for PEA 1 and PEA 2 structure). Possibly, the L-LA oligomeric segment interacts with the amide-containing segment leading to a decrease in its crystallinity, which is reflected in a lowering of the melting temperature (from $140{ }^{\circ} \mathrm{C}$ to $\mathrm{ca} 130{ }^{\circ} \mathrm{C}$ ). For PEA 1, the endothermic peak at ca $85{ }^{\circ} \mathrm{C}$ has vanished in the second heating run, but a new peak is observed at $c a 46{ }^{\circ} \mathrm{C}$. This temperature can be attributed to L-LA oligomeric segment melting. Thus, it seems that in PEA 1 two crystalline phases exist: one attributed to the L-LA oligomeric segment and the other to the segment containing the amide linkages. This has been observed in block PEAs containing poly(L-lactide) segments. ${ }^{2}$ PEA 2, in turn, presents a complex melting behaviour in the second heating run, where two additional peaks at $45{ }^{\circ} \mathrm{C}$ and $67{ }^{\circ} \mathrm{C}$ are identified. At this point, a possible explanation for this fact can be related to the rearrangement of PEG and L-LA oligomeric segments within the PEA 2 chain during the cooling run, leading to a crystalline structure that subsequently melts in the second heating run.

The glass transition temperature $\left(T_{\mathrm{g}}\right)$ of the PEAs was determined by MDSC and DMTA. The results obtained are presented in Table 5 (see Supporting Information for MDSC and DMTA traces in the glass transition zone, Fig. S2). PEA 1 and PEA 2 show ca 10 ${ }^{\circ} \mathrm{C}$ difference between $T_{\mathrm{g}}$ values determined by MDSC and DTMA, which can be ascribed to differences in definition of the glass transition, as mentioned elsewhere. ${ }^{28}$ The difference obtained for PEA 3 could be due to the high crystallinity of the sample, which makes an accurate $T_{\mathrm{g}}$ determination difficult in the two methods.

Nevertheless, a remarkable decrease in $T_{\mathrm{g}}$ with the introduction of the L-LA oligomers is observed, suggesting that these segments act as a plasticizer of the PEA chain. When the L-LA oligomeric segment is included in the PEAs, the crystalline structure can be disrupted, leading to more amorphous materials that present 


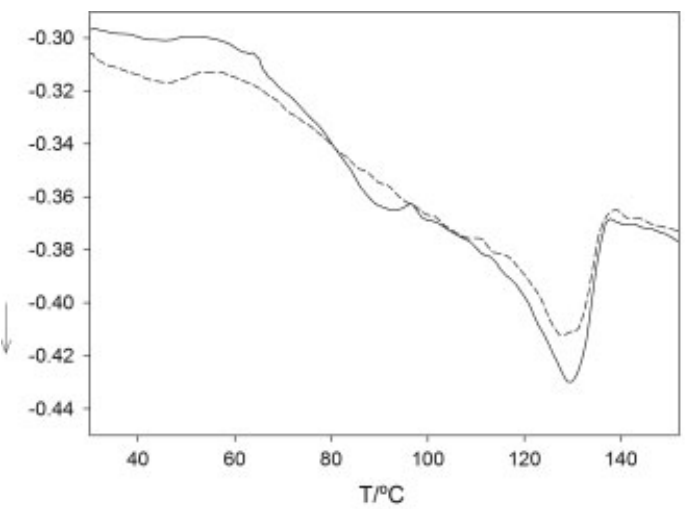

PEA 1

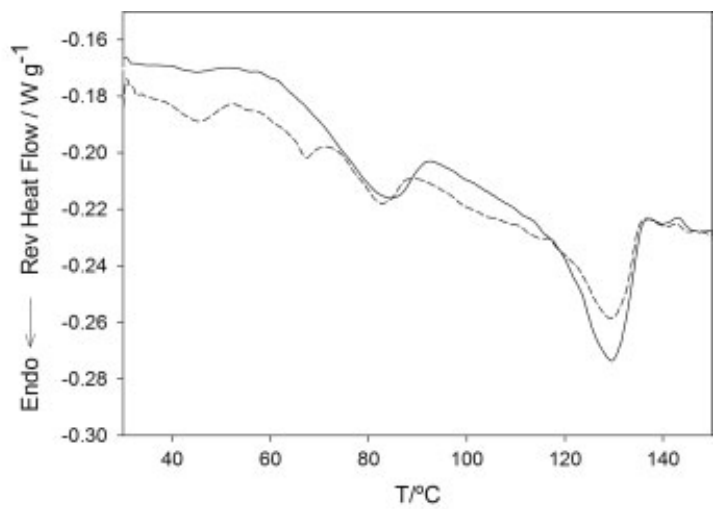

PEA 2

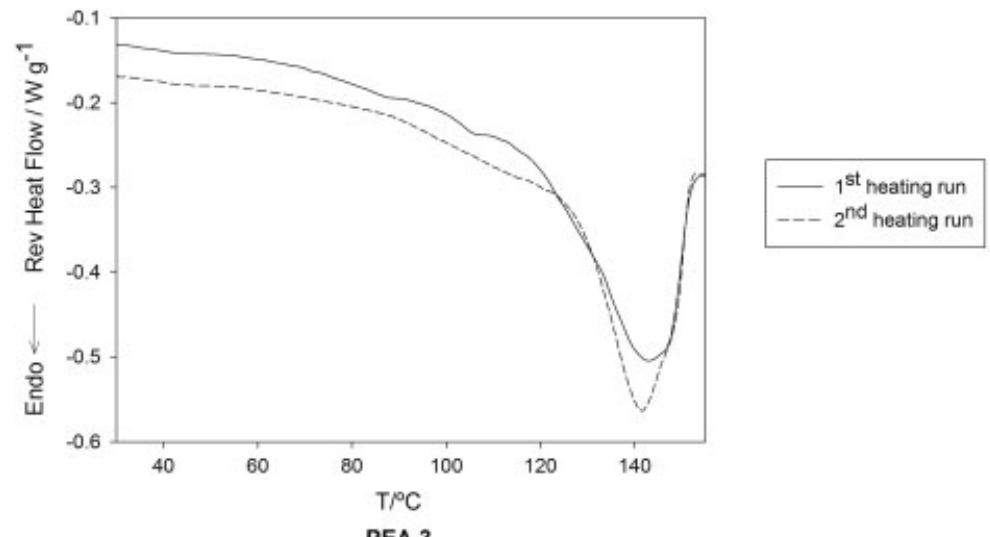

Figure 6. MDSC curves of the PEAs $\left(2{ }^{\circ} \mathrm{C} \mathrm{min}-1\right.$ modulation period: $60 \mathrm{~s}$; temperature modulation of $\pm 0.40{ }^{\circ} \mathrm{C}$; for clarity, only reverse heat flow is presented).

\begin{tabular}{|lcc|}
\hline $\begin{array}{l}\text { Table 5. } \\
\text { DMTA }\end{array}$ & $T_{\mathrm{g}}$ values for the synthesized PEAs obtained by MDSC and \\
Poly(ester amide) & $T_{\mathrm{g}, \mathrm{MDSC}}\left({ }^{\circ} \mathrm{C}\right)$ & $T_{\mathrm{g}, \mathrm{DMTA}}\left({ }^{\circ} \mathrm{C}\right)$ \\
\hline PEA 1 & -5.7 & 4.6 \\
PEA 2 & -16.8 & -4.5 \\
PEA 3 & 98.3 & 41 \\
\hline
\end{tabular}

higher chain flexibility. PEA 2 presents a lower $T_{\mathrm{g}}$ due to the higher content of ether linkages. PEA 3, in turn, presents a higher $T_{\mathrm{g}}$ value and its $T_{\mathrm{g}}$ peak is broader than those observed for PEA 1 and PEA 2 , which is indicative of a higher crystalline content. In addition, the difference in molecular weight of the PEAs also contributes to the difference in $T_{\mathrm{g}}$ values.

\section{CONCLUSION}

Novel PEAs from glycine and L-LA were prepared using an easy, catalyst-free and fast polymerization method. A diamine based on glycine and DEG was used for the first time. The structural analysis proved the success of the synthesis and thermal analysis showed that building blocks can be used to tune the thermal properties. Considering the potential of $\alpha$ amino acid/ $\alpha$-hydroxy acid PEAs in the biomaterials area, the synthetic route reported here can be used as proof of concept for the synthesis of new PEAs with tailor-made properties by using different $\alpha$-amino acids (with specific side groups). The required optimization studies are being done and will be reported elsewhere.

\section{ACKNOWLEDGEMENTS}

A.C. Fonseca acknowledges Fundação para a Ciência e Tecnologia, Grant SFRH/BD/41305/2007. The ${ }^{1} \mathrm{H}$ NMR data were obtained from Rede Nacional de RMN in the University of Coimbra, Portugal. The Varian VNMRS $600 \mathrm{MHz}$ spectrometer is part of the National NMR Network and was purchased in the framework of the National Program for Scientific Re-equipment (contract REDE/1517/RMN/2005, with funds from POCI 2010 (FEDER) and Fundação para a Ciência e Tecnologia (FCT)).

\section{SUPPORTING INFORMATION}

Supporting information may be found in the online version of this article.

\section{REFERENCES}

1 Lu DD, Ren ZL, Zhou TH, Wang SF and Lei ZQ, J Appl Polym Sci 107: 3638-3643 (2008).

2 Luckachan GE and Pillai CKS, J Polym Sci Polym Chem 44: 3250-3260 (2006).

3 Nair LS and Laurencin CT, Prog Polym Sci 32: 762-798 (2007).

4 Feng Y, Lu J, Behl M and Lendlein A, Macromol Biosci 10: 1008-1021 (2010).

5 Ohya Y, Matsunami H, Yamabe E and Ouchi T, J Biomed Mater Res A 65A: 79-88 (2003). 
6 Ohya Y, Nakai T, Nagahama K, Ouchi T, Tanaka S and Kato K, J Bioact Compat Polym 21: 557-577 (2006).

7 Karimi P, Rizkalla AS and Mequanint K, Materials 3: 2346-2368 (2010).

8 Armelin E, Paracuellos N, Rodriguez-Galan A and Puiggali J, Polymer 42: $7923-7932$ (2001).

9 Montane J, Armelin E, Asin L, Rodriguez-Galan A and Puiggali J, J Appl Polym Sci 85: 1815-1824 (2002).

10 Dijkstra PJ and Feijen J, Macromol Symp 153: 67-76 (2000).

11 Feng YK and Guo JT, Int J Mol Sci 10: 589-615 (2009).

12 Katsarava R, Ochkhikdze N, Tugushi D and Gomurashvili ZD, AABBPoly(depsipeptide) biodegradable polymers and methods of use. US Patent, US2010/0040664 A1, USA (2010).

13 Odian G, Principles of Polymerization. John Wiley \& Sons, Hoboken, NJ (2004).

14 D'Angelo S, Galletti P, Maglio G, Malinconico M, Morelli P, Palumbo R et al, Polymer 42: 3383-3392 (2001).

15 Katsarava R, Beridze V, Arabuli N, Kharadze D, Chu CC and Won CY, J Polym Sci Polym Chem 37: 391-407 (1999).

16 Guo K and Chu CC, Biomacromolecules 8: 2851-2861 (2007).

17 Hiltunen K, Harkonen M, Seppala JV and Vaananen T, Macromolecules 29: 8677-8682 (1996)

18 Hiltunen K and Seppala JV, J Appl Polym Sci 67: 1017-1023 (1998).
19 Paredes N, Rodriguez-Galan A and Puiggali J, J Polym Sci Polym Chem 36: 1271-1282 (1998).

20 Asin L, Armelin E, Montane J, Rodriguez-Galan A and Puiggali J, J Polym Sci Polym Chem 39: 4283-4293 (2001).

21 Desimone V, Maglio G, Palumbo R and Scardi V, J Appl Polym Sci 46: 1813-1820 (1992).

22 Khan W, Muthupandian S, Farah S, Kumar N and Domb AJ, Macromol Biosci 11: 1625-1636 (2011).

23 Knight DK, Gillies ER and Mequanint K, Biomacromolecules 12: 2475-2487 (2011).

24 Fonseca AC, Coelho JFJ, Valente JFA, Correia TR, Correia IJ, Gil MH, Simöes PN, submitted for publication in Macromolecular Bioscience.

25 van Bennekom ACM, Willemsen PAAT and Gaymans RJ, Polymer 37: 5447-5459 (1996).

26 Garg P, Keul H, Klee D and Möller M, Macromol Chem Phys 210: 1754-1765 (2009).

27 Serrano PJM, van de Werff BA and Gaymans RJ, Polymer 39: 83-92 (1998).

28 Wunderlich B, Thermal Analysis of Polymeric Materials. Springer, Heidelberg (2005). 